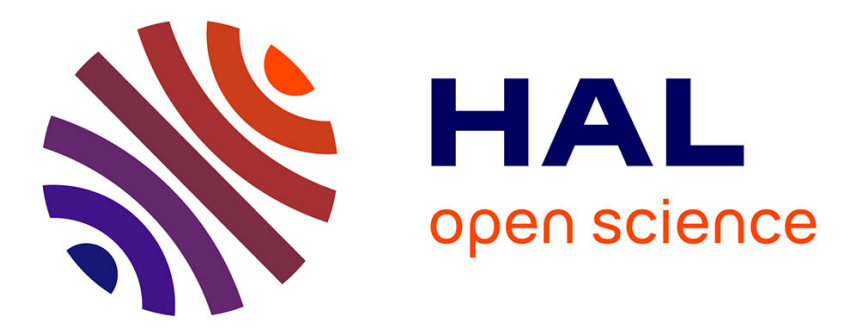

\title{
Treatment of Primary Central Nervous System Lymphoma in Immunocompetent Patients
}

Ytel Garcilazo-Reyes, Agusti Alentorn, Alberto Duran-Peña, Khe Hoang-Xuan, Caroline Houillier

\section{- To cite this version:}

Ytel Garcilazo-Reyes, Agusti Alentorn, Alberto Duran-Peña, Khe Hoang-Xuan, Caroline Houillier. Treatment of Primary Central Nervous System Lymphoma in Immunocompetent Patients. Current Treatment Options in Neurology, 2019, 21 (8), pp.39. 10.1007/s11940-019-0578-x . hal-02385142

\section{HAL Id: hal-02385142 https://hal.sorbonne-universite.fr/hal-02385142}

Submitted on 28 Nov 2019

HAL is a multi-disciplinary open access archive for the deposit and dissemination of scientific research documents, whether they are published or not. The documents may come from teaching and research institutions in France or abroad, or from public or private research centers.
L'archive ouverte pluridisciplinaire HAL, est destinée au dépôt et à la diffusion de documents scientifiques de niveau recherche, publiés ou non, émanant des établissements d'enseignement et de recherche français ou étrangers, des laboratoires publics ou privés. 


\title{
Treatment of Primary Central Nervous System Lymphoma in immunocompetent patients
}

Garcilazo-Reyes Ytel $^{1}$, Alentorn Agusti ${ }^{1,2}$, Duran-Pena Alberto ${ }^{1}$, Hoang-Xuan Khê $\hat{e}^{1,2}$, Houillier Caroline ${ }^{1,2}$

1-AP-HP, Sorbonne Université, Department of Neurology-2, Groupe Hospitalier Pitié Salpêtrière, Paris, France

2- LOC network for PCNSL

Corresponding author: Khê Hoang-Xuan, MD, PhD

Department of Neurology-2, Groupe Hospitalier Pitié Salpêtrière, Paris, France

e-mail: khe.hoang-xuan@aphp.fr

Keywords- Primary Central Nervous System lymphoma chemotherapy, radiation therapy, Methotrexate, autologous stem cell transplantation, immunotherapy, rituximab, ibrutinib, imids.

\begin{abstract}
Purpose of review: This review focus on the findings of recent randomized prospective trials evaluating new therapeutic options for primary central nervous system lymphoma (PCNSL) in first line treatment and on the most promising novel agents.

Recent findings: The current standard treatment of newly diagnosed PCNSL has long been depending on high dose methotrexate (HD-MTX) based polychemotherapy followed by whole brain radiotherapy (WBRT). Recent randomized trials have provided evidence that high dose chemotherapy with ASCT is a valuable alternative option to WBRT as consolidation after induction HD-MTX based chemotherapy. For the elderly, cumulative studies confirm that chemotherapy alone as initial treatment is the best approach in this frail population in order to reduce chemoradiation neurotoxicity. If the role of rituximab needs to be further investigated, novel agents such as imids and ibrutinib have showed promising drugs to be incorporated in innovative combination treatment.
\end{abstract}

Summary: The role of WBRT, at least at conventional dose, is declining in first line treatment.in favor of intensive consolidation chemotherapy with or without ASCT and possibly maintenance chemotherapy in the elderly. Despite their rarity, it has been shown that ambitious randomized trials in PCNSL are feasible thanks to collaborative networks.

\section{Introduction}

PCNSLs are rare non-Hodgkin lymphomas of the diffuse large B-cell (DLBC) type that involve the brain, eyes, leptomeninges, or spinal cord, without any systemic lymphoma. Because of the multifocal and infiltrative nature of PCNSL, its well-known chemosensitivity and radiosensitivity, and the lack of randomized trials addressing specifically the interest of cytoreductive surgery [1][2], the role of surgery is widely limited to a stereotactic biopsy for establishing a histopathologic diagnosis [3]. The current standard 
treatment of newly diagnosed PCNSL has long been depending on high dose methotrexate (HD-MTX) based polychemotherapy followed by WBRT[3,4]. However, despite a high rate of remission after first line treatment, relapses remain frequent and the overall outcome is poor. In addition, the patients, especially the elderly, are exposed to a high risk of treatment-related neurocognitive dysfunctions and deleterious impact on quality of life [5]. In order to optimize treatment, several approaches have been proposed such as immunochemotherapy with rituximab, high dose chemotherapy consolidation regimens with autologous stem cell transplantation (HCT-ASCT), reduced dose WBRT in order to minimize the delayed neurotoxicity of combined treatment, maintenance chemotherapy, especially for fragile patients who would not be eligible for WBRT or ASCT. Here, we reviewed recent published and ongoing controlled clinical trials addressing these issues. We also focused on the most promising new agents that would contribute to improve the management of PCNSL.

\section{Induction immunochemotherapy with rituximab in first line treatment}

Induction chemotherapy aims to obtain rapidly the highest complete response (CR) rate before consolidation. If HD-MTX is considered as the standard drug for PCNSL treatment, a polychemotherapy is recommended in order to increase objective response rate and to circumvent chemoresistance. Drugs crossing the blood brain barrier, such as procarbazine, temozolomide, ifosfamide, etoposide, carmustine, lomustine,thiotepa, cytarabine, have been the most frequently used in combination with HD-MTX in PCNSL. In addition, cumulative evidence suggesting that immunotherapy with anti-CD20 rituximab might improve outcome as it does in systemic DLBC lymphomas despite its poor CNS penetration, has led to its incorporation into many induction immunochemotherapy regimens. Hence, several single arm phase II trials have reported encouraging results combining MTX, rituximab and alkylating agents with post-induction CR rates of 60$66 \%$, and 2-year progression free survival (PFS) ranging from 57 to $79 \%$ according to subsequent consolidation treatment [6-9]. However, trials evaluating induction (immuno)chemotherapy combinations with control group are scarce. The international IELSG32 trial is a two sequential randomized phase II study, which randomly assigned 227 patients in three induction chemotherapy arms. The chemotherapy regimens included: (i) HD-MTX and cytarabine only as control group [10], (ii) the same regimen combined with rituximab, or (iii) the same regimen combined with rituximab and thiotepa (named MATRix schedule). Patients with responsive or stable disease underwent a second randomization to consolidation with WBRT versus HCT-ASCT. The results of the first randomization demonstrated that the addition of rituximab alone and thiotepa-rituximab to MTX-cytarabine increased CR rates (CR rate: 31\% and 49\% respectively) compared with the control arm (CR rate: 23\%). Although the trial was not designed for comparison, the CR rate increased significantly only when rituximab was combined with thiotepa over the control group, addition of rituximab alone was associated with a significant higher objective response rate (ORR: CR + Partial Response, PR) compared with the control arm (ORR: 73\% versus 53\%). Interestingly, the addition of thiotepa to rituximab further improved ORR rate (86\%) and outcome (hazard ratio [HR] for overall survival $0 \cdot 41,95 \%$ CI $0 \cdot 25-1 \cdot 68$ ) over the control group [11]. However, efficacy of rituximab in PCNSL could 
not be confirmed in the HOVON 105/ALLG NHL 24 randomized phase III trial (conducted in the Netherlands, Australia, and New Zealand), which randomly assigned 200 patients with newly diagnosed PCNSL to receive rituximab in addition to HD-MTX based induction chemotherapy (including carmustine, etoposide) or the induction chemotherapy alone. After induction, all responding patients received consolidation chemotherapy with cytarabine, and only those aged younger than 60 years received additional WBRT. The authors failed to find any improvement with rituximab in terms of event-free survival (EFS), which was the primary endpoint ( [HR] 1.00; 95\% CI 0.70-1.43), of PFS (HR : 0.77; 95\% CI 0.52-1.13), overall survival (OS) (HR:0.93; 95\% CI :0.59-1.44) and post induction chemotherapy CR rate (30\% versus $36 \%$ ) compared to chemotherapy alone. Surprisingly, in a subgroup analysis by age, patients aged $<60$ years seemed to benefit from addition of rituximab in terms of PFS (HR 0.48; 95\% CI: 0.26-0.88), EFS (HR: 0.56; 95\%CI 0.31-1.01), and OS (HR 0.59; 95\%CI: 0.28-1.24) [12]. As WBRT consolidation was proposed only to younger patients, a possible interaction with treatment has been raised. However, this was an unplanned post-hoc analysis that has to be interpreted with caution. Taking into account these two latter prospective studies with opposite conclusions, the identification of the patients who would benefit from rituximab warrant to be further investigated by clinical trials specifically dedicated to this issue and by age group.

\section{Consolidation strategies in first line treatment}

The role of WBRT as consolidation after HD-MTX based induction polychemotherapy has been challenged by cumulative studies in the recent years [3,13], thus encouraging the evaluation of alternative strategies. The rationale behind the use of HCT-ASCT in PCNSL patients resides in the administration of maximum tolerable doses of cytostatic drugs, to reach therapeutic concentrations in the CNS to eradicate microscopic residual disease, overcoming drug resistance mediated by blood brain barrier. HCT-ASCT, has showed its efficacy and feasibility in patients with relapsed PCNSL[14,15]. Prospective studies have reported encouraging results with this approach as consolidation in first line treatment using different conditioning regimens [6,16-18]. Although comparison between conditioning regimens is difficult in the absence of controlled study, high-dose thiotepa-based conditioning regimens (carmustine-thiotepa, thiotepa-cyclophosphamide-busulfan) seem the most efficient whereas results with BEAM regimen appeared disappointing [19]. Comparison of the high dose chemotherapy with ASCT approach with the chemo-radiotherapy combination as first line treatment has been investigated by two phase II randomized trials with PFS at 2 years as primary endpoint and with prospective psychometric testing follow-up [20,21].

The IELSG32 trial cited above randomly assigned, in its second randomization stage, 118 eligible patients out of 227 patients after induction chemotherapy to receive WBRT (36 Gy with 1.8 Gy / fraction, with the addition of a 9 Gy tumor-bed boost in patients in partial response) or ASCT (using a carmustine-thiotepa conditioning regimen). In the PRECIS trial conducted by the ANOCEF-GOELAMS intergroup, 97 eligible patients out of 140 patients enrolled in the study who have received an induction MTX based immunochemotherapy (rituximab, MTX, carmustine, etoposide, cytarabine), were assigned to receive WBRT 
(40 Gy with 2 Gy / fraction) or ASCT (using thiotepa-cyclophosphamide-busulfan). Both trials met the predetermined efficacy threshold in both arms and confirmed the efficacy of WBRT and ASCT as consolidation. In addition, they demonstrated a better preservation of cognitive functions after ASCT, while, as expected, a higher hematological toxicity than in those who received WBRT. Treatment-related death occurred with ASCT only (3\% in IELSG32 and 11\% in PRECIS). Although both trials were not designed to allow direct comparison of the consolidation therapies, the IELSG32 trial, reported no significant differences in 2-year PFS between WBRT and ASCT, while in the PRECIS trial, the efficacy endpoints tended to favor the ASCT arm with a 2-year PFS rates of $63 \%$ (95\% CI 49-81) and 87\% (95\% CI 77-98) in the WBRT and ASCT arms, respectively. Despite common objectives and endpoints, the IELSG32 and PRECIS trials differed by several relevant aspects, including their designs, treatment protocols and patient selection. Particularly, in the PRECIS trial the upper age limit was lower ( $<60$ years), the randomization was done at trial registration, the induction chemotherapy regimen was homogeneous, the WBRT total dose was higher and the conditioning regimen could be considered as more intensive. Altogether, the two latter randomized trials provide evidence to support the role of ASCT as a valuable alternative option to WBRT, at least at conventional dose. However, longer follow-up is needed to evaluate more precisely the potential delayed consequences on neurocognition and quality of life of the ASCT consolidation [20,21].

The MSKCC has reported encouraging results both in terms of efficacy and neurotoxicity in a prospective

single arm study using a low-dose WBRT (23.4 Gy in 13 fractions) as consolidation following an induction immunochemotherapy including high-dose MTX, procarbazine, rituximab, and cytarabine[7]. This approach is currently evaluated in a randomized phase II trial (RTOG-1114) comparing the MSKCC regimen with or without reduced-dose WBRT has been conducted (NCT01399372). The trial is now completed, and the results are awaited. On the basis of the efficacy of an induction regimen combining rituximab, HD-MTX, and temozolomide (RMT), followed by consolidation with a non myeloablative chemotherapy comprising highdose etoposide and cytarabine [22,23], the CALGB group is conducting a randomized phase II (NCT01511562) in which patients receive RMT induction chemotherapy followed by consolidation with high dose etoposide-cytarabine or thiotepa- carmustine followed by ASCT.

\section{Treatment of the elderly}

Treatment of the elderly, who constitute the majority of PCNSL immunocompetent patients, is challenging as they represent a highly vulnerable population. Given the high risk of severe neurotoxicity following chemoradiation therapy. There is nowadays a wide agreement to consider HD-MTX-based chemotherapy with deferral or elimination of WBRT as the treatment of choice [3,24-26]. Controlled trials dedicated to this age group are scarce. To date the only one, is randomized phase II trial devoted to patients aged over 60 treated with either MPV-A regimen (MTX, procarbazine, vincristine, cytarabine) or MTX plus temozolomide without consolidation WBRT has been conducted by the ANOCEF-GOELAMS intergroup [27]. Both chemotherapy regimens met the predetermined efficacy threshold confirming prospectively their efficacy. 
MPV-A treated patients showed better outcomes in terms of CR rate, median PFS, and OS, compared with MTX plus temozolomide, although these differences did not reach level of significance. Both regimens were associated with manageable toxicity and psychometric and quality of life follow-up showed significant improvements over time, confirming the value of this approach in the elderly. However, in spite of high objective response rates remission were short-lasting and the OS were poor. Maintenance treatment is a potential valuable alternative strategy to prolong disease control. An ongoing phase III trial conducted by the French LOC network address this question (NCT02313389). Patients who have achieved a CR after an induction chemotherapy (rituximab, MPV-A) are randomly assigned to receive a maintenance chemotherapy combining rituximab, MTX and temozolomide versus observation. In selected elderly PCNSL patients, consolidation with high-dose chemotherapy with ASCT [28] or with high-dose cytarabine [29] using chemotherapy regimens adapted to age have been showed to be feasible in retrospective studies and warrant to be further investigated in controlled prospective trials.

\section{New agents: biotherapies}

With the current management of PCNSL, about one third of patients are refractory to first-line treatment and the majority of the patients will eventually relapse. The prognosis is poor after salvage therapy, overall, the median PFS and OS after recurrence were 2.2 months (range, 0-29.6) and 3.5 months (0-29.6), respectively, in a large population-based study, elderly remaining the group with the worse outcome [30]. To date, there is no standard treatment however, HCT-ASCT is the best available option in selected candidates for refractory/relapsing PCNSL, with 2-year PFS of 62\% (35\%- 85\%), median PFS up to 25.8 months and 2-year OS of $63 \%(51 \%-74 \%)$ [15][14]. New active agents are needed to improve salvage treatment but also to strengthen the first line chemotherapy regimens. Promising results have been reported with ibrutinib and imids.

\section{Ibrutinib}

Ibrutinib is a selective inhibitor of Bruton tyrosine kinase (BTK), targeting B cell receptor (BCR) signaling which is particularly active in PCNSL with recurrent mutations altering BCR subunit CD79B and MYD88. These mutations have been found in PCNSL at higher rate than in other extracerebral DLBCL for which it has been shown an efficacy of ibrutinib. Ibrutinib has been investigated in PCNSL as single agent in three clinical trials with promising efficacy $[31,32,33]$. Grommes et al reported the results of a phase I study of ibrutinib given at escalating dose to up to $840 \mathrm{mg}$ daily in patients with refractory and relapsing primary or secondary CNS lymphomas. Response was observed in $77 \%$ of 13 patients with PCNSL with a PFS of 4.6 months [31]. The LOC network has conducted a phase II trial enrolling 52 patients with refractory and relapsed PCNSL (including primary vitreoretinal lymphoma) using 560mg daily dosed ibrutinib (NCT02542514). Intent to treat response rate was observed in 52\% of cases after 2 cycles, with a PFS of 4.8 months and OS: 19 
months[33]. Lionakis et al in a phase I of the NCI, reported a partial response rate of $83 \%$ two weeks after ibrutinib introduction, in a series of 18 patients suffering from newly diagnosed and relapsing PCNSL [32]. The specific contribution of ibrutinib on the final outcome is difficult to analyze, as a wide chemotherapy combination (DA-TEDDi-R) was rapidly added to ibrutinib. In this latter study, several unexpected cases of severe pulmonary and CNS aspergillosis, were reported raising the possible role of the treatment combination that could have exacerbated the ibrutinib induced fungal immune surveillance impairment. More recently, Grommes et al. showed in a phase $1 \mathrm{~b}$ clinical trial that the sequential combination of ibrutinib (at dose of 560 or $840 \mathrm{mg}$ ) with HD-MTX and rituximab followed by maintenance ibrutinib in patients with refractory/relapsing CNS lymphoma was active and well tolerated [34], laying the bases for future comparative trials evaluating ibrutinib based combination treatment.

\section{Imids}

Imids including lenalidomide and pomalidomide are thalidomide-derived agents with antiproliferative and immunomodulatory properties which have shown antilymphoma activity, likely in the activated $\mathrm{B}$ cell (ABC) subtype of DLBCL. Lenalidomide as single agent has been used in patients with relapsing PCNSL, with 2 patients achieving a CR in a small retrospective cohort [35]. The activity was confirmed in a phase I with 9 objective response out of 14 relapsed/refractory PCNSL treated with lenalidomide monotherapy (15 mg on a 21day cycle) [36]. A phase II study conducted by the LOC network and the LYSA evaluated lenalidomide (20 mg on a 21day cycle) in combination with rituximab in 50 relapsed /refractory PCNSL and primary vitreoretinal lymphoma (PVRL) and found an ORR of $35 \%$. Responding patients were proposed a maintenance with lenalidomide alone and the median PFS and OS were 7.8 and 17.7 months respectively [37]. Pomalidomide is a third-generation imid that has shown a good CNS penetration [38]. In a phase I study, Tun et al. assessed the efficacy of pomalidomide with dexamethasone in relapsed/refractory PCNSL and PVRL patients [39]. The ORR reported was 48\% and a median PFS of 5.3 months and 9 months for responders. In regard of their good safety profiles, imids would be potential interesting agents to improve response rates by incorporating induction regimens but also in the setting of maintenance treatment. Indeed, encouraging results with prolonged PFS and OS have been reported in a small cohort of 13 elderly patients (median age: 77 years; range 70-86), who have been treated with low dose lenalidomide (5-10 mg/day on a 21day cycle) after achieving an objective response to a HD-MTX based chemotherapy as induction [40].

\section{Conclusion}

The management of patients suffering from PCNSL has been substantially improved in the past decade as a result of better knowledge of treatment strategies. The role of WBRT as consolidation is clearly declining in favor of high dose chemotherapy approaches with or without ASCT; and, for the elderly, there is now a large consensus to avoid its use in first line treatment in order to reduce the risk of neurotoxicity. Further efforts should focus on increasing the remission rate and prolonging their duration with a curative aim. In this setting, 
the role of rituximab remains unclear and further studies are needed to identify the patients who will benefit from this treatment. Hope come clearly from new targeted agents and immunomodulatory therapies which have shown promising results and already incorporated in innovative combination treatment evaluated in ongoing trials. Prospective trials evaluating the role of immune therapy with checkpoint inhibitors and anti CD19 CAR T cells in PCNSL are also awaited[41,42]. Despite their rarity, it has been shown that ambitious randomized trials in PCNSL are feasible thanks to collaborative networks at the national and international level. Lessons from previous trials pointed out the importance to build adequate and robust designs for the future trials to allow firm conclusions. Elderly, representing a growing population that requires a specific management, should be investigated likely in dedicated trials.

\section{References}

1. Weller M, Martus P, Roth P, Thiel E, Korfel A. Surgery for primary CNS lymphoma? Challenging a paradigm. Neuro Oncol. 2012;14:1481-4.

2. Labak CM, Holdhoff M, Bettegowda C, Gallia GL, Lim M, Weingart JD, et al. Surgical Resection for Primary Central Nervous System Lymphoma: A Systematic Review. World Neurosurg. 2019;

3. Hoang-Xuan K, Bessell E, Bromberg J, Hottinger AF, Preusser M, Rudà R, et al. Diagnosis and treatment of primary CNS lymphoma in immunocompetent patients: guidelines from the European Association for Neuro-Oncology. Lancet Oncol. 2015;16:e322-332.

4. DeAngelis LM, Yahalom J, Thaler HT, Kher U. Combined modality therapy for primary CNS lymphoma. J Clin Oncol. 1992;10:635-43.

5. van der Meulen M, Dirven L, Habets EJJ, van den Bent MJ, Taphoorn MJB, Bromberg JEC. Cognitive functioning and health-related quality of life in patients with newly diagnosed primary CNS lymphoma: a systematic review. Lancet Oncol. 2018;19:e407-18.

6. Omuro A, Correa DD, DeAngelis LM, Moskowitz CH, Matasar MJ, Kaley TJ, et al. R-MPV followed by high-dose chemotherapy with TBC and autologous stem-cell transplant for newly diagnosed primary CNS lymphoma. Blood. 2015;125:1403-10.

7. Morris PG, Correa DD, Yahalom J, Raizer JJ, Schiff D, Grant B, et al. Rituximab, methotrexate, procarbazine, and vincristine followed by consolidation reduced-dose whole-brain radiotherapy and cytarabine in newly diagnosed primary CNS lymphoma: final results and long-term outcome. J Clin Oncol. 2013;31:3971-9.

8. Rubenstein JL, Hsi ED, Johnson JL, Jung S-H, Nakashima MO, Grant B, et al. Intensive chemotherapy and immunotherapy in patients with newly diagnosed primary CNS lymphoma: CALGB 50202 (Alliance 50202). J Clin Oncol. 2013;31:3061-8.

9. Swinnen LJ, O’Neill A, Imus PH, Gujar S, Schiff D, Kleinberg LR, et al. Phase II study of rituximab given in conjunction with standard chemotherapy in primary central nervous system lymphoma (PCNSL): a trial of the ECOG-ACRIN cancer research group (E1F05). Oncotarget. 2018;9:766-73.

10. Ferreri AJM, Reni M, Foppoli M, Martelli M, Pangalis GA, Frezzato M, et al. High-dose cytarabine plus high-dose methotrexate versus high-dose methotrexate alone in patients with primary CNS lymphoma: a 
randomised phase 2 trial. Lancet. 2009;374:1512-20.

11. Ferreri AJM, Cwynarski K, Pulczynski E, Ponzoni M, Deckert M, Politi LS, et al. Chemoimmunotherapy with methotrexate, cytarabine, thiotepa, and rituximab (MATRix regimen) in patients with primary CNS lymphoma: results of the first randomisation of the International Extranodal Lymphoma Study Group-32 (IELSG32) phase 2 trial. Lancet Haematol. 2016;3:e217-227.

** randomized trial comparing different induction chemo-immunotherapies in PCNSL with the best results obtained with a combination of rituximab, thiotepa, MTX and cytarabine

12. Bromberg JEC, Issa S, Bakunina K, Minnema MC, Seute T, Durian M, et al. Rituximab in patients with primary CNS lymphoma (HOVON 105/ALLG NHL 24): a randomised, open-label, phase 3 intergroup study. Lancet Oncol. 2019;20:216-28.

**Phase III trial failing to show a benefit to add rituximab to hd MTX based induction chemotherapy

13. Thiel E, Korfel A, Martus P, Kanz L, Griesinger F, Rauch M, et al. High-dose methotrexate with or without whole brain radiotherapy for primary CNS lymphoma (G-PCNSL-SG-1): a phase 3, randomised, noninferiority trial. Lancet Oncol. 2010;11:1036-47.

14. Soussain C, Hoang-Xuan K, Taillandier L, Fourme E, Choquet S, Witz F, et al. Intensive chemotherapy followed by hematopoietic stem-cell rescue for refractory and recurrent primary CNS and intraocular lymphoma: Société Française de Greffe de Moëlle Osseuse-Thérapie Cellulaire. J Clin Oncol. 2008;26:25128.

15. Kasenda B, Ihorst G, Schroers R, Korfel A, Schmidt-Wolf I, Egerer G, et al. High-dose chemotherapy with autologous haematopoietic stem cell support for relapsed or refractory primary CNS lymphoma: a prospective multicentre trial by the German Cooperative PCNSL study group. Leukemia. 2017;31:2623-9.

16. Alnahhas I, Jawish M, Alsawas M, Zukas A, Prokop L, Murad MH, et al. Autologous Stem-Cell Transplantation for Primary Central Nervous System Lymphoma: Systematic Review and Meta-analysis. Clin Lymphoma Myeloma Leuk. 2019;19:e129-41.

17. Illerhaus G, Kasenda B, Ihorst G, Egerer G, Lamprecht M, Keller U, et al. High-dose chemotherapy with autologous haemopoietic stem cell transplantation for newly diagnosed primary CNS lymphoma: a prospective, single-arm, phase 2 trial. Lancet Haematol. 2016;3:e388-397.

18. Cho H, Chang JH, Kim YR, Kim S-J, Chung H, Park H, et al. The role of upfront autologous stem cell transplantation in high-risk younger patients with primary central nervous system lymphoma. Br J Haematol. 2016;174:444-53.

19. Abrey LE, Moskowitz CH, Mason WP, Crump M, Stewart D, Forsyth P, et al. Intensive methotrexate and cytarabine followed by high-dose chemotherapy with autologous stem-cell rescue in patients with newly diagnosed primary CNS lymphoma: an intent-to-treat analysis. J Clin Oncol. 2003;21:4151-6.

20. Houillier C, Taillandier L, Dureau S, Lamy T, Laadhari M, Chinot O, et al. Radiotherapy or Autologous Stem-Cell Transplantation for Primary CNS Lymphoma in Patients 60 Years of Age and Younger: Results of the Intergroup ANOCEF-GOELAMS Randomized Phase II PRECIS Study. J Clin Oncol. 2019;JCO1800306.

** Randomized trial favoring the use of high dose chemotherapy with ASCT over WBRT as consolidation in younger patients 
21. Ferreri AJM, Cwynarski K, Pulczynski E, Fox CP, Schorb E, La Rosée P, et al. Whole-brain radiotherapy or autologous stem-cell transplantation as consolidation strategies after high-dose methotrexate-based chemoimmunotherapy in patients with primary CNS lymphoma: results of the second randomisation of the International Extranodal Lymphoma Study Group-32 phase 2 trial. Lancet Haematol. 2017;4:e510-23.

** Second randomization of the large phase II trial comparing consolidation strategies of WBRT and high dose chemotherapy with ASCT and providing evidence of the efficacy of both approaches

22. Rubenstein JL, Hsi ED, Johnson JL, Jung S-H, Nakashima MO, Grant B, et al. Intensive chemotherapy and immunotherapy in patients with newly diagnosed primary CNS lymphoma: CALGB 50202 (Alliance 50202). J Clin Oncol. 2013;31:3061-8.

23. Birsen R, Willems L, Pallud J, Blanc E, Burroni B, Legoff M, et al. Efficacy and safety of high-dose etoposide cytarabine as consolidation following rituximab methotrexate temozolomide induction in newly diagnosed primary central nervous system lymphoma in immunocompetent patients. Haematologica. 2018;103:e296-9.

24. Hoang-Xuan K, Taillandier L, Chinot O, Soubeyran P, Bogdhan U, Hildebrand J, et al. Chemotherapy alone as initial treatment for primary CNS lymphoma in patients older than 60 years: a multicenter phase II study (26952) of the European Organization for Research and Treatment of Cancer Brain Tumor Group. J Clin Oncol. 2003;21:2726-31.

25. Fritsch K, Kasenda B, Schorb E, Hau P, Bloehdorn J, Möhle R, et al. High-dose methotrexate-based immuno-chemotherapy for elderly primary CNS lymphoma patients (PRIMAIN study). Leukemia. 2017;31:846-52.

26. Gavrilovic IT, Hormigo A, Yahalom J, DeAngelis LM, Abrey LE. Long-term follow-up of high-dose methotrexate-based therapy with and without whole brain irradiation for newly diagnosed primary CNS lymphoma. J Clin Oncol. 2006;24:4570-4.

27. Omuro A, Chinot O, Taillandier L, Ghesquieres H, Soussain C, Delwail V, et al. Methotrexate and temozolomide versus methotrexate, procarbazine, vincristine, and cytarabine for primary CNS lymphoma in an elderly population: an intergroup ANOCEF-GOELAMS randomised phase 2 trial. Lancet Haematol. 2015;2:e251-259.

* A randomized phase II trial dedicated to elderly evaluating two chemotherapy regimen MPV-A regimen and MTX-temozolamide regimen without WBRT, with psychometric and quality of life follow-up confirming the value of this approach in this fragile population

28. Schorb E, Fox CP, Fritsch K, Isbell L, Neubauer A, Tzalavras A, et al. High-dose thiotepa-based chemotherapy with autologous stem cell support in elderly patients with primary central nervous system lymphoma: a European retrospective study. Bone Marrow Transplant. 2017;52:1113-9.

29. Houillier C, Ghesquières H, Chabrot C, Soussain C, Ahle G, Choquet S, et al. Rituximab, methotrexate, procarbazine, vincristine and intensified cytarabine consolidation for primary central nervous system lymphoma (PCNSL) in the elderly: a LOC network study. J Neurooncol. 2017;133:315-20.

30. Langner-Lemercier S, Houillier C, Soussain C, Ghesquières H, Chinot O, Taillandier L, et al. Primary CNS lymphoma at first relapse/progression: characteristics, management, and outcome of 256 patients from 
the French LOC network. Neuro-oncology. 2016;18:1297-303.

31. Grommes C, Pastore A, Palaskas N, Tang SS, Campos C, Schartz D, et al. Ibrutinib Unmasks Critical Role of Bruton Tyrosine Kinase in Primary CNS Lymphoma. Cancer Discov. 2017;7:1018-29.

* Mutational landscape of PCNSL and trial using ibrutinib in refractory/relapsed PCNSL.

32. Lionakis MS, Dunleavy K, Roschewski M, Widemann BC, Butman JA, Schmitz R, et al. Inhibition of B Cell Receptor Signaling by Ibrutinib in Primary CNS Lymphoma. Cancer Cell. 2017;31:833-843.e5.

*Ibrutinib as monotherapy and in combination in PCNSL

33. Soussain C, Choquet S, Houillier C, Bijou F, Houot R, Boyle E, et al. Study of Ibrutinib in Patients With Relapsed or Refractory Primary Central Nervous Lymphoma or Intraocular Lymphoma. Results of the primary end point of the LOC phase II study from the LYSA and the French LOC network. Hematological Oncology. 2017;35(S2):72

34. Grommes C, Tang SS, Wolfe J, Kaley TJ, Daras M, Pentsova EI, et al. Phase 1b trial of an ibrutinib-based combination therapy in recurrent/refractory CNS lymphoma. Blood. 2019;133:436-45.

* Phase I MTX and ibrutinib combination therapy

35. Houillier C, Choquet S, Touitou V, Martin-Duverneuil N, Navarro S, Mokhtari K, et al. Lenalidomide monotherapy as salvage treatment for recurrent primary CNS lymphoma. Neurology. 2015;84:325-6.

36. Rubenstein JL, Geng H, Fraser EJ, Formaker P, Chen L, Sharma J, et al. Phase 1 investigation of lenalidomide/rituximab plus outcomes of lenalidomide maintenance in relapsed CNS lymphoma. Blood Adv. 2018;2:1595-607.

37. Ghesquieres H, Chevrier M, Laadhari M, Chinot O, Choquet S, Moluçon-Chabrot C, et al. Lenalidomide in Combination with Intravenous Rituximab (REVRI) in Relapsed/Refractory Primary CNS Lymphoma or Primary Intraocular Lymphoma: a Multicenter Prospective "Proof of Concept" Phase II Study of the French Oculo-Cerebral Lymphoma (LOC) Network and the Lymphoma Study Association (LYSA). Ann Oncol. 2019; 30:621-628

* Phase II study evaluating lenalidomide with rituximab in r/r PCNSL and PVRL

38. Li Z, Qiu Y, Personett D, Huang P, Edenfield B, Katz J, et al. Pomalidomide Shows Significant Therapeutic Activity against CNS Lymphoma with a Major Impact on the Tumor Microenvironment in Murine Models. PLoS One. 2013; 8(8):e71754

39. Tun HW, Johnston PB, DeAngelis LM, Atherton PJ, Pederson LD, Koenig PA, et al. Phase 1 study of pomalidomide and dexamethasone for relapsed/refractory primary CNS or vitreoretinal lymphoma. Blood. 2018;132:2240-8.

40. Vu K, Mannis G, Hwang J, Geng H, Rubenstein JL. Low-dose lenalidomide maintenance after induction therapy in older patients with primary central nervous system lymphoma. Br J Haematol. 2019; version of record online February 3, 2019.

41. Nayak L, Iwamoto FM, LaCasce A, Mukundan S, Roemer MGM, Chapuy B, et al. PD-1 blockade with nivolumab in relapsed/refractory primary central nervous system and testicular lymphoma. Blood. 2017;129:3071-3.

42. Abramson JS, McGree B, Noyes S, Plummer S, Wong C, Chen Y-B, et al. Anti-CD19 CAR T Cells in 
CNS Diffuse Large-B-Cell Lymphoma. N Engl J Med. 2017;377:783-4. 